\title{
Histoire et philosophie des sciences : traduire les mots-clefs des concepts scientifiques
}

\author{
Sylvie Nony \\ professeur agrégée de Physique \\ Doctorante à Paris VII, unité d'accueil du CHSPAM ${ }^{1}$ \\ Recherches menées à la bibliothèque de l'Institut des Études \\ Dominicaines du Caire
}

L'étude de la transmission des savoirs scientifiques et philosophiques du monde grec au monde arabe a permis de montrer les liens profonds et la continuité des concepts entre les deux civilisations. Longtemps oublié de l'histoire occidentale des connaissances, l'héritage arabo-musulman reprend peu à peu la place qui lui est due. L'analyse des apports spécifiques de celui-ci n'est cependant pas toujours aisée comme le montre l'exemple de la théorie du rhôpê-mayl-intentio-impetus que nous allons examiner ici. La problématique de l'héritage, que ce soit des grecs aux arabes, ou des arabes aux latins, ne rend pas compte à elle seule de tous les mouvements d'idées scientifiques et philosophiques. Dans une certaine mesure même, elle les masque et l'exemple de ce qu'on appelle fréquemment «le mayl des arabes» est à cet égard assez significatif. Il révèle pour une part un problème technique de traduction en histoire des sciences, mais, au-delà, il révèle aussi la façon dont sont appréhendés les concepts scientifiques historiques. 


\section{I/ Introduction}

Les travaux d'Ibn Sīnā puis ceux d'Abū 1-Barakāt al-Bag̉dādī² ont intégré l'apport de Jean Philopon (VIe siècle AC) à la théorie d'Aristote sur le mouvement des projectiles. L'impulsion (rhopê) de Jean Philopon est devenue mayl - mot dont il est l'exact pendant en arabe - dans les textes d'Ibn Sīnā et a été conceptualisé par celui-ci comme une force acquise (quwwa mustafāda) permettant d'expliquer pourquoi un projectile continue sa trajectoire lorsqu'il se sépare de son moteur (le lanceur). Cette théorie sera reprise dans le monde latin par Jean Buridan et les terminalistes parisiens sous le vocable d'impetus ou celui d'inclinatio, terme qui coïncide sémantiquement avec celui de mayl qui est un substantif formé sur le verbe māla, pencher, incliner. En dépit du terme commun de mayl une étude comparée de l'utilisation de ce concept dans la Physique d'Ibn Sīnā et celle d'Abū 1-Barakāt al-Bağdādī montre des différences colossales, alors qu'ils partent tous les deux de la même définition : "chaque mouvement provient d'un mayl que vérifie l'impulsion ('indifā') de la chose qui se tient devant le mobile et son besoin d'une force par laquelle elle résiste 〈au mobile> $»^{3}$. C'est que les conceptions du temps, de l'espace physique, et au-delà, celles de la connaissance, qui forment le cadre de leurs études respectives sont radicalement différentes. Or, comme l'affirmaient Duhem et Quine, ce sont «collectivement que les énoncés affrontent le tribunal de l'expérience ». On ne peut faire parler les mots et notamment les mots d'une science qu'en montrant leur pouvoir explicatif au sein d'une théorie admise comme un corps organisé. Nous souhaiterions montrer ici que la théorie du mayl est loin d'être unique et que ce sont les contradictions mêmes entre ses 
différentes acceptions - plutôt que leur unité - qui peuvent avoir fécondé les bouleversements scientifiques ultérieurs, notamment la physique galiléenne. La chose est banale en sciences : un mot unique peut masquer de véritables controverses théoriques. Les mots relativité, éther, onde... et tant d'autres ont eu ce sort. Mais la traduction d'un tel concept par un mot translittéré comme cela est l'usage - voire la règle ${ }^{4}$ - fait prendre un risque encore plus grand d'une lecture monolithique et c'est là une difficulté supplémentaire. Comme le remarque Pierre Pellegrin, helléniste et traducteur de la Physique d'Aristote: "Traduire des textes grecs anciens pose deux sortes de problèmes. Des problèmes «techniques » dont certains sont propres au fait de traduire le grec, le grec scientifique et le grec d'Aristote. Mais il y a aussi les problèmes liés au «statut»du texte que l'on traduit : à quelle entreprise le traducteur prend-il part quand il traduit des textes «scientifiques» grecs et plus particulièrement aristotéliciens?».

A quelle entreprise le traducteur des textes arabes sur la physique des projectiles prend-il part? C'est la question que nous aimerions aborder ici.

\section{II/ Les amonts du mayl}

Les deux auteurs arabes sur lesquels nous nous focalisons, Ibn Sīnā (m 1037) et Abū l-Barakāt (m 11525), -tous les deux dans une large mesure pouvant être qualifiés d'aristotéliciens-, partent d'une même définition, mot pour mot, du concept de mayl, définition élaborée $^{6}$ par le premier d'entre eux. Mais il est très instructif d'étudier de près comment ce concept est mis à l'épreuve dans un $1 \leqslant r$ 
exercice de pensée classique (depuis Aristote et jusqu'à la fin du moyen âge) : la «quies media». Il s'agit de trancher la question suivante : lorsqu'un objet est jeté en l'air à la verticale, vers le haut, et qu'il enchaîne son mouvement ascendant d'un mouvement descendant vers le sol, existe-t-il un instant de repos (quies) entre (media) ces deux mouvements ? On pourrait s'étonner qu'une question pareille ait pu faire couler tant d'encre pendant une vingtaine de siècles. C'est qu'elle met en jeu, comme on va le voir, des concepts bien plus fondamentaux même s'ils ne sont pas toujours explicités.

Lorsque Jean Philopon (m 575 environ), au sein de l'école de philosophie d'Alexandrie, a élaboré son concept de rhopê, il l'a fait dans le cadre d'une physique qui, si elle doit encore beaucoup à Aristote, contient une rupture essentielle concernant la physique du mouvement: celle-ci peut être conçue en séparant les mobiles du milieu dans lequel ils évoluent. Jean Philopon élabore une physique des projectiles qui peut se concevoir dans le vide c'est-à-dire dans un espace absolu, débarrassé de l'exigence de contact permanent entre un mobile et son moteur comme l'exigeait Aristote. Cette rupture permet à Jean le Grammairien de penser le mouvement dans le vide comme un passage à la limite de l'évolution du mouvement de ce mobile dans un milieu de plus en plus ténu. Ce terme grec de rhopê ne donnera pas lieu, à cette époque et dans cette langue, à autant d'échafaudages théoriques que le mayl puisque, cinquante ans après la mort de Jean Philopon, l'Égypte est conquise par les arabes et que c'est dans cette langue que se fera la transmission de l'apport du Grammairien. Un héritage dont les arabes se revendiquent avec une telle conviction que plusieurs de leurs historiens ont retardé la date de sa mort au-delà de 


\section{Sylvie Nony}

la conquête musulmane de l'Egypte par 'Amru Ibn 'As (640), pour faire de ce dernier un protecteur du philosophe (en butte aux autorités chrétiennes) ${ }^{7}$.

Lorsque Ibn Sīnā reprend le rhopê de Philopon, il est dans une toute autre démarche. Il n'est pas question pour lui d'admettre le vide, et encore moins de remettre en question le dogme de la nécessité d'un moteur à tout mouvement (il est donc très loin du principe d'inertie qu'on lui attribue parfois). Comme l'a montré Ahmed Hasnawi dans une étude très détaillée de cette théorie ${ }^{8}$, il cherche à remédier à une faiblesse majeure de la théorie d'Aristote qui tente d'expliquer la poursuite du mouvement de la flèche loin de l'arc par la mise en mouvement de l'air qu'elle chasse devant elle et qui se rassemble derrière elle : pourquoi derrière, s'interrogent tous les commentateurs et pourquoi admettre pour l'air, ce qu'on refuse à la flèche, à savoir le pouvoir de conserver la force motrice acquise du lanceur ? Ibn Sīnā remédie ainsi avec son mayl conçu comme une «force acquise », à une faiblesse patente de la théorie d'Aristote, sans rien égratigner de l'édifice causal de celui-ci. En particulier le monde reste plein, chaque effet a une cause et la notion de force à distance est proscrite.

Lorsque Abū 1-Barakāt al-Baġdādī, ardent lecteur de Jean Philopon, reprend sa conception de l'espace débarrassé de tout milieu et donc vide, ainsi que ses conceptions de l'infini, il a une toute autre démarche même s'il introduit son propos sur le mayl par une définition reprise à la lettre de celle du maître Avicenne (voir citation plus haut). Il en fait un outil pour construire une continuité causale et un continuum dans l'évolution des paramètres du mouvement qui permettra, en fait, d'envisager plus tard une mathématisation de celuici.

$1 \leq 0$ 


\section{II/ L'épreuve de la quies media}

Le mouvement vertical d'un projectile vers le haut se décompose en deux parties (voire trois si on ajoute le repos controversé) : le mouvement vers le haut, contre nature, appelé par nos deux auteurs mouvement violent, et le mouvement de chute appelé mouvement naturel.

Ce qui suit est le commentaire d'une présentation diaporama faite lors de la conférence du Caire dont certaines images sont données en illustration.

\section{$\underline{2.1 \text { Le mouvement violent ascendant }}$}

La théorie de la quwwa mustafāada d'Ibn Sīnā permet en apparence d'internaliser le mayl qasry (inclination violente) dans le corps, et résout certaines contradictions de la théorie d'Aristote concernant le mouvement de l'air supposé en faire un moteur. Mais ce mayl est considéré comme une cause du mouvement. Or la cessation d'un effet correspond toujours à la privation de sa cause : ce mayl doit être épuisé par le milieu, ce qui a pour corollaire le fait qu'il continuerait indéfiniment dans le vide. Fort heureusement le vide n'existe pas et l'absence de mouvement infini est une preuve supplémentaire de sa non-existence. Le milieu est donc indispensable pour comprendre le mouvement ascendant. Il n'y a donc pas internalisation complète de la cause du mouvement dans le mobile.

Le mayl violent d'Abū l-Barakāt al-Bag̉dādī a presque le statut d'un effet : sa cause est le lanceur que le mobile a quitté. Cet effet est contrecarré par celui du mayl naturel, seule force présente pendant tout le mouvement. Il peut donc y avoir pour Abū l-Barakāt une 


\section{Sylvie Nony}

vitesse vers le haut alors que la force est vers le bas, ce qui est une audace de pensée d'importance. Le milieu n'a aucun rôle décisif si ce n'est celui d'ajouter un ralentissement supplémentaire à la vitesse vers le haut.

Faire de l'histoire des sciences au futur antérieur consisterait à dire qu'Ibn Sīnā s'approche du principe d'inertie (puisque son mayl ne s'épuise pas de façon intrinsèque) et qu'Abū 1-Barakāt s'en éloigne (puisque son mayl qasry s'épuise) alors que le point de rupture est davantage dans la conception de l'espace (toujours forcément plein et aristotélicien pour le premier, et pouvant être vide pour le second) ainsi que dans la distinction fondamentale entre le concept de force et celui de vitesse initiale communiquée par le lanceur: Abū 1-Barakāt affirme que la seule force qui est présente tout au long du mouvement est en fait, le poids du corps.

\subsection{Le mouvement naturel descendant}

Pour Ibn Sīnā, arrivé au sommet de sa trajectoire, le mobile n'a plus de mayl qasry puisque celui-ci s'annule quand son «but» est atteint. C'est donc toujours dans le cadre d'une physique aristotélicienne qu'Ibn Sīnā réfléchit en donnant le rôle de cause à ce qui relève de l'avenir du mouvement (le sommet de la trajectoire). Alors et seulement alors, une nouvelle « cause » peut entrer en jeu : le mayl naturel. Les effets de celle-ci ne peuvent démarrer que lorsque le premier mayl est épuisé : deux mayls différents ne peuvent coexister dans un même corps.

Pour Abū 1-Barakāt le mayl naturel était déjà là pendant la montée. Il le démontre avec l'expérience (de pensée) du lancer de $1 \leqslant \vee$ 
deux pierres de masses différentes vers le haut : la plus grosse se déplace plus lentement. C'est bien la preuve que deux mayls peuvent coexister dans un même corps. Le mayl naturel prend le dessus au fur et à mesure où la vitesse vers le haut diminue. Ce mayl qasri est même la cause d'un mouvement descendant plus lent quand il fait suite à une montée 9 . Son effet contraire à celui du poids induit un début de descente plus lent. Mais il y a continuité complète entre les deux mouvements. Après avoir fait dire à Ibn Sīnā : «N'écoute pas celui qui dit que les deux mayls sont réunis. Comment serait-il possible que l'impulsion dans une direction, ou sa nécessité, soit dans une chose en acte et <dans le même temps> que l'exclusion de cette impulsion le soit aussi ? Ne pense jamais que la pierre lancée vers le haut a un mayl en elle jusqu'en bas mais seulement un principe venant de sa matière qui rend possible qu'advienne un tel mayl si l'obstacle cesse et qu'il est vaincu, de la même façon que dans l'eau il y a une force et un principe, et le froid survient dans sa substance, si l'obstacle cesse et qu'il est vaincu, comme tu l'as appris ${ }^{10}$, Abū 1-Barakāt enchaîne : "D'une part le ralentissement de la montée est dû̀ l'affaiblissement du mayl violent et au rapprochement ${ }^{11}$ du mayl naturel ${ }^{12}$ qui y résiste. D'autre part la chute est <encore $>$ faible parce qu'on en est au début de la force du mayl naturel. En tant qu'il est contrôlé par le mayl naturel, il contient l'anéantissement de ce qui reste comme puissance au mayl violent au fur et à mesure, jusqu'à ce qu'il cesse ; alors sa résistance cesse <aussi>. C'est pour cela que le mayl naturel devient de plus en plus vigoureux à la fin, alors que le mayl violent était le plus vigoureux au début. ${ }^{13}{ }^{»}$ 


\subsection{Le repos intermédiaire}

Puisqu'il y a disjonction des causes du mouvement violent d'une part et naturel d'autre part, il y a forcément pour Ibn Sīnā un temps d'arrêt entre le montant et le descendant.

Pour Abū l-Barakāt al-Bağdādī il y a continuité totale et donc aucun temps d'arrêt ne s'impose : la quies media n'existe pas comme il en fait la démonstration au chapitre 24. Il s'agit de la démonstration théorique qu'une même et unique cause peut avoir des effets contraires et que ces deux contraires peuvent se suivre en continuité, sans « repos intermédiaire »: Parlant de lui à la troisième personne, «l'Unique de son Temps » comme il se surnommait, raconte qu'il «a pris une règle, a fait en son centre un trou puis il a fait passer dans ce trou un fil auquel était suspendu un plomb. Ensuite il a placé l'extrémité finale du fil à l'extrémité fermée de la règle dans une rainure et il a fait déplacer le fil en ligne droite dans ce trait du début de la règle à sa fin en passant près du trou : tant que ce fil se dirige vers le trou, le fil à plomb est conduit vers le bas jusqu'à ce qu'il parvienne à une extrémité, lorsqu'il est proche 〈du trou〉. Ensuite il remonte en poursuivant son mouvement lorsque <le fil> s'éloigne $<d u$ trou $>$ pour se diriger vers l'autre extrémité $<$ de la règle $>$... Lorsque le fil est près du milieu, les deux mouvements contraires sont alors indispensables au fil à plomb dans la poursuite du chemin d'un mouvement unique rectiligne, dans une trajectoire unique provenant d'un moteur unique, atteint dans un temps unique. Et quand alors, aurait lieu le repos?"

Les graphes qui suivent cherchent à traduire ces différences en ce qui concerne trois grandeurs aujourd'hui représentables et $1 \leqslant 9$ 
mesurables (mais qui étaient loin de l'être à l'époque) :

- le temps dont Abū 1-Barakāt se refusait à donner une représentation spatiale étant donné qu'il a pour particularité de ne jamais changer de sens d'écoulement,

- la vitesse qui dans la physique aristotélicienne s'apparente systématiquement à une vitesse moyenne, alors qu'Abū 1-Barakāt va faire intervenir une vitesse que l'on qualifierait aujourd'hui d'instantanée (représentée en pointillé)

- une force dont l'intensité est souvent évaluée par les deux philosophes, en comparant son effet avec celui d'une autre sur le même corps dans les mêmes conditions (représentée en trait plein) 

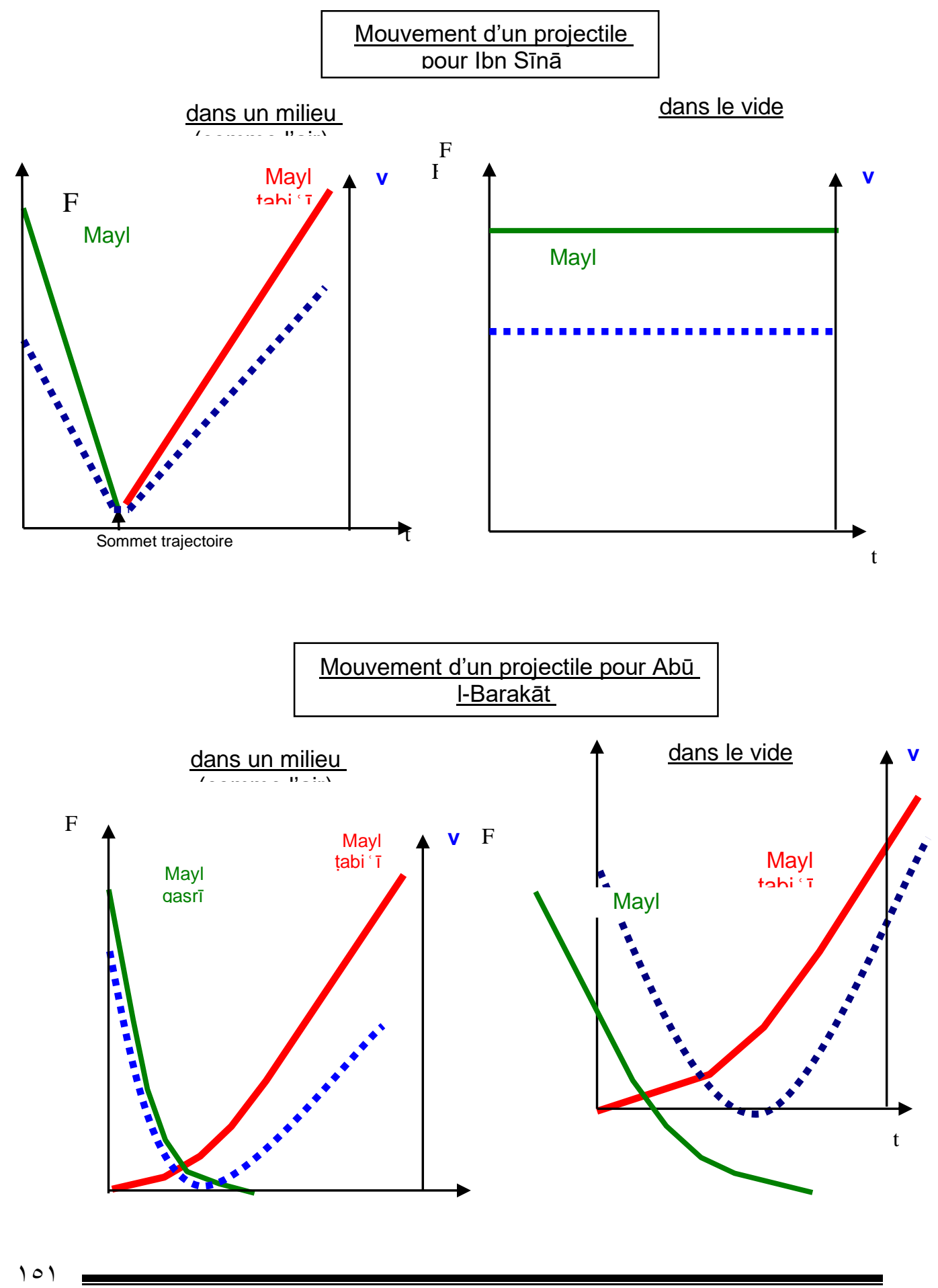
- Histoire et philosophie des sciences : traduire les mots-clefs des concepts scientifiques

\section{III/ Un mayl peut en cacher un autre}

Les différences entre les deux théories évoquées sautent aux yeux. Elles sont aussi importantes et de même nature que celles entre l'impetus du De Motu de Galilée et l'impetus de la fin de sa vie : un mayl qui se ramène à son effet peut être évalué (comme l'est une vitesse). Il peut co-exister avec une force constante (le poids) dont on comprend mal pourquoi elle surviendrait en haut de la trajectoire.

Ce renversement causal permet à Abū 1-Barakāt al-Bağdādī de conceptualiser pour la première fois l'accélération de la chute des graves pour laquelle Aristote dans le De Caelo n'avait trouvé d'autre explication que l'augmentation de la gravité lorsque le corps se rapproche du centre de la Terre. Comme l'a fait remarquer Alexandre Koyré ${ }^{14}$, Aristote était prisonnier de son axiome « une force constante doit produire un mouvement de vitesse constante $»$.

Ibn Sīnā dans les Mubahathat ${ }^{15}$ avait déjà évoqué l'idée d'une sommation de mayls pour éviter d'avoir recours à l'explication d'Aristote mais il maintenait l'idée que cette sommation de mayl se faisait « en vue de ce centre de la Terre ». Abū 1-Barakāt affirme lui que c'est à la hauteur de chute déjà parcourue que la vitesse est proportionnelle. Ce qui, même si une telle relation débouche sur une solution exponentielle au lieu de la parabole que tout le monde connaît, délivre l'analyse du mouvement de sa cause finale : le paramètre qui gouverne le mouvement dépend de son passé mais plus de son avenir. Il est géométrique et donc mathématisable.

La continuité entre les concepts de rhopê/mayl/impetus mérite donc d'être interrogée : au-delà de la nécessaire recréation propre à chaque traduction/transmission, il y a des bouleversements conceptuels que les termes ne laissent pas entrevoir. Sauf à vouloir cantonner la civilisation arabo-musulmane dans un rôle de traducteur -plus ou moins créatif- il nous 
faut approfondir les changements complets de paradigme que l'usage d'un seul mot ne laisse pas entrevoir, montrer la pluralité de ce concept et la fertilité des contradictions soulevées.

La précaution de conserver le mot dans la langue d'origine lorsqu'il n'a pas d'équivalent dans la langue de traduction n'apporte donc pas ipso facto une plus grande précision scientifique ni un plus grand respect de la pensée contenue dans le texte source. Elle contribue plus souvent à mettre le mot « sous cloche » et à le chosifier.

Comment à l'inverse rendre vivant ce terme ? Comment traduire toutes les polysémies d'un terme technique sans alourdir son emploi de longues périphrases? La science moderne essaie d'affecter à chaque concept un mot et un seul, quitte à fabriquer des mots de toute pièce. $\mathrm{Ne}$ devrions nous pas en faire autant plus souvent, quitte à fabriquer un mot pour chaque mayl ? La poésie des commentaires s'en ressentirait un peu, mais les analyses y gagneraient en précision. 
- Histoire et philosophie des sciences : traduire les mots-clefs des concepts scientifiques

\section{Notas}

1 Centre d'Histoire des Sciences et des Philosophies Arabes et Médiévales

2 Le kitāb as- šifă' pour Ibn Sīnā, et le kitāb al-mu atabar pour Abū l-Barakāt

3 Kitāb al-mu 'atabar, II, chap 24, p 95 et kitāb as- šifă' , as--Samā ' at-țabī 'i , p 141

4 C'est ainsi que dans "Règles pour éditions et traductions de textes arabes », éd. Les Belles Lettres 1953, Blachère et Sauvaget indiquent concernant les termes techniques:

Règle 111: ... " il convient de rendre en français tout ce qui peut être traduit sans déformer la pensée de l'auteur ».

Règle 115: "les mots qui ne sauraient être rendus en français qu'au moyen d'une périphrase alourdissant le texte seront simplement transcrits ....et imprimés en italique ; ils ne devront pas être pliés aux règles de la grammaire française ".

5 Date approximative, sujette à discussion

6 al-Fārābī dans des textes perdus aujourd'hui, semble avoir lui aussi, défini un mayl dans des termes peut-être similaires.

7 La légende de Jean Philopon chez les Arabes, par Armand ABEL, in Correspondances d'Orient (Bruxelles) 10 (Acta Orientalia Belgica 63/64)

8 Ahmed Hasnaoui, La dynamique d'Ibn Sīnā, In Études sur Avicenne, Les Belles Lettres, 1984

9 C'est une assertion admise depuis Aristote sans plus de démonstration : un mobile qui est en chute libre depuis une certaine altitude $\mathrm{z}$ tombe plus vite que le même mobile en chute depuis la même altitude $\mathrm{z}$ mais dont le mouvement fait suite à un mouvement ascendant.

10 Abū l-Barakāt al-Bag̉dādī, kitāb al-mu 'atabar, chap 24 p96

11 Muqāraba (rapprochement) dans le ms courant et muqārina (mașḍar de qārana, être uni, associé, simultané) dans le ms سـ ce qui, compte tenu de la note qui suit, revient au même.

12 Plus exactement du rapprochement de la prise de contrôle du mouvement par le mayl naturel

13 Op.cit. p 100 et 101

14 Koyré A., La loi de la chute des corps, Revue Philosophique 1937, mai-août

15 Cité par Ahmed Hasnawi dans La dynamique d'Ibn Sinnā, op. cit. 


\section{Bibliographie}

Outre les ouvrages cités en note de l'article :

1. Aristote, Physique. Traduction de Pierre Pellegrin, GF Flammarion, 2000.

2. Abū 1-Barakāt al-Bag̉dādī, Al kitāb al-mu atabar fì al-ḥikma al-ilāhya, ed. Ḥīderābād, $1357(\mathrm{H})$

3. Hasnaoui A., Le statut catégorial du mouvement chez Avicenne, in Cahiers du Mideo , 2004, La définition du mouvement dans la Physique du Shifa' d'Avicenne, in Arabic Sciences and Philosophy 11, 2001.

4. Pinès S., Studies in Abū l-Barakāt al-Bàgdādī Physics and Metaphysics, in Collected Works of Shlomo Pines, The Hebrew University, Jerusalem, 1979

5. Sorabji R., John Philoponus. In: Philoponus and the Rejection of Aristolelian Science. Duckworth, London, 1991.

6. Duhem P., Le Système du Monde, Hermann Paris, 1916, 1988.

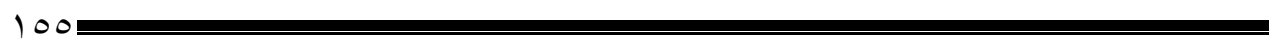

